\title{
High Efficiency and Problems of Chemiluminescence Assay-Detected Aldosterone-To-Renin Ratio in Practical Primary Aldosteronism Screening
}

\author{
Wenbin Lin, ${ }^{1}$ Yuzhe Li, ${ }^{2}$ Dubo Chen, ${ }^{1}$ Zhenrong Yao, ${ }^{1}$ Hongxu Xu, ${ }^{1}$ Yonghong Chen, ${ }^{3}$ \\ Jiahao Xiao, ${ }^{4}$ Pinning Feng $\left(\mathbb{D}^{1}{ }^{1}\right.$ and Wenjia Gan $\mathbb{1}^{1}$ \\ ${ }^{1}$ Department of Clinical Laboratory, The First Affiliated Hospital of Sun Yat-Sen University, Guangzhou, China \\ ${ }^{2}$ Department of Clinical Laboratory, The Third Affiliated Hospital of Sun Yat-Sen University, Guangzhou, China \\ ${ }^{3}$ Department of Clinical Laboratory, The Sixth People's Hospital of Longgang District, Shenzhen, China \\ ${ }^{4}$ Reproductive Medicine Center, The Third Affiliated Hospital of Sun Yat-Sen University, Guangzhou, China
}

Correspondence should be addressed to Pinning Feng; fengpn@mail.sysu.edu.cn and Wenjia Gan; wenjiagan@hotmail.com

Received 29 October 2019; Revised 8 July 2020; Accepted 16 July 2020; Published 27 August 2020

Academic Editor: Tomohiro Katsuya

Copyright (๑) 2020 Wenbin Lin et al. This is an open access article distributed under the Creative Commons Attribution License, which permits unrestricted use, distribution, and reproduction in any medium, provided the original work is properly cited.

Primary aldosteronism is a main cause of secondary hypertension which can be effectively treated. The screening test for primary aldosteronism is benefit for minimizing damage to the patient. In the previous retrospective study, we obtained the optimal cutoff value of aldosterone-to-renin ratio detected by chemiluminescence assay, a newly developing method, and prompted its high efficiency in primary aldosteronism screening in upright position. In this study, we want to evaluate its efficiency in practical work. We used this ratio to continuously screen 238 patients, and 58 patients were finally diagnosed with primary aldosteronism. We found it had $86.13 \%$ accuracy rate in the upright position compared with the final clinical diagnosis. False negative and positive rates were $13.79 \%$ and $13.89 \%$. Diagnostic sensitivity and specificity were $86.21 \%$ and $86.11 \%$, which are slightly different from results in our previous study. False negative rate can be improved by combining the aldosterone-to-renin ratio with aldosterone concentration. We also found impaired glucose tolerance may be a reason for high false positive rate. Besides, chemiluminescence assay may be interfered in aldosterone detection. Although it has some shortcomings, chemiluminescence assay-detected aldosterone-to-renin ratio is a highly effective index for screening primary aldosteronism in practice.

\section{Introduction}

Primary aldosteronism (PA), which accounts for $5 \%$ to $20 \%$ morbidity in resistant hypertension in different reports [1-3], is a main cause of secondary hypertension [4]. It is caused by excess aldosterone secretion from one or both of adrenal glands. Excess aldosterone not only causes hypertension through sodium and water retention but also directly induces damage to vital organs, like the heart, kidney, and vasculature through inflammation, fibrosis, and tissue remodeling. Thus, PA patients are more likely to have arrhythmia, ventricular hypertrophy, cerebral infarction, renal insufficiency, etc. PA can be effectively treated by blocking aldosterone or hyperplastic adrenal gland resection [5]. So, effectively discovering disease has great significance for PA patients.

The aldosterone-to-renin ratio (ARR) is widely used to screen out PA from secondary hypertension. Results from a multicenter study show that ARR application raises 10-15 times PA diagnosis rate [2] and has been encouraged by the Endocrine Society Guideline [6]. In recent decades, chemiluminescence assay (CLIA) is applied for aldosterone and renin detection, which is a higher safety profile than the previously used radioimmunoassay (RIA) [7, 8]. Although it is recommended in aldosterone and renin detection, CLIA is not extensively used in PA diagnosis, because of the controversies in the cutoff value and diagnostic efficiency of 
CLIA-detected ARR [7, 9-11]. Therefore, more evidence on these two areas should be collected.

In our previous study, we determined the optimal cutoff value and diagnostic efficiency of CLIA-detected ADRR in PA screening by retrospective analysis [12]. We found that CLIA-detected ADRR has the highest diagnostic efficiency, especially specificity and positive-predictive value, when the cutoff value is 28 in the upright position. But, the ARR cutoff value for PA screening is recommended as 30 or more in other studies [13-15], which is larger than our cutoff value. We wonder whether our cutoff value is suitable for the practical PA screening. In this study, we evaluate the efficiency of our ADRR cutoff value in PA screening through a high-quality perspective study. Our results confirm the conclusion in the previous study. Meanwhile, we discover some interfering factors in practical use. Our study may attribute to the widespread application of CLIA-detected ADRR in PA screening.

\section{Materials and Methods}

2.1. Patients. We sequentially collected data from patients with refractory hypertension to the First Affiliated Hospital of Sun Yat-sen University (Guangzhou, China) between October 2018 and March 2019. Refractory hypertension was regarded as hypertension without remission after enough treatment according to the current guidelines. ADRRs of eligible patients were detected immediately after admission. Final diagnosis was recorded after the patient leaving the hospital. PA diagnosis was according to the guideline about PA diagnosis and treatment, which was published by the American Association of Clinical Endocrinologists in 2016 [6] and PA diagnosis standards in China. Briefly, PA diagnosis was required to satisfy following conditions: (1) typical clinical features, like hypertension and hypokalemia; (2) adrenal hyperplasia or adenoma in imaging; (3) histopathology; and (4) approval test confirmation (aldosterone concentration over $100 \mathrm{pg} / \mathrm{ml}$ in the saline infusion test or renin concentration decreased less than $30 \%$ in the captopril test). The case included in the following study should at least have a complete ADRR in the upright position and a clear PA or non-PA diagnosis.

2.2. Sample Collection and Detection. This study was approved by the Medical Ethics Committee of the hospital, and the requirement of written informed consent was remitted by the Medical Ethics Committee. According to guidelines, patients were asked to withdraw all antihypertensive drugs at least 2 weeks before blood collection. During drug withdrawal, the patients were carefully looked after. Drugs without influence on aldosterone and renin concentrations, like diltiazem, doxasozine, and verapamil, were used as escape medication in case of severe discomfort and/or extreme blood pressure $(\geq 180 / 110 \mathrm{mmHg})$. Blood sample was collected after a full night's sleep $(>8 \mathrm{~h})$. After that, patients were asked to stand or walk for $2 \mathrm{~h}$ and seat for $15 \mathrm{~min}$. Then, blood sample in the upright position was collected before 9 : $00 \mathrm{AM}$ on the same day. The specimen was collected in
EDTA-K $\mathrm{K}_{2}$ anticoagulant tube. Plasma was separated by centrifugation at room temperature, $3,000 \mathrm{~g}$ for $5 \mathrm{~min}$. CLIA kits and detecting instrument (Antu Biotech Co., LTD, Zhengzhou, China) were used to detected aldosterone and renin concentrations in plasma, which followed the manufacturer's instruction. The analytical imprecision of CLIAdetected aldosterone and renin both was less than 5\%. The measuring ranges of CLIA-detected aldosterone and renin were $10-1000 \mathrm{pg} / \mathrm{ml}$ and $4-500 \mathrm{pg} / \mathrm{ml}$. The reference intervals of aldosterone and renin in our lab were $40-310 \mathrm{pg} / \mathrm{ml}$ and 4-38 pg/ml, respectively. Corresponding aldosterone and renin concentrations were used to calculate ADRR. ADRR $>28$ was considered as positive for PA screening [12].

2.3. Statistical Analysis. SPSS v22.0 (IBM) was used in the whole statistical analysis. Quantitative data were expressed as mean value \pm standard deviation (SD) or indicated. Final clinical diagnosis was considered as the gold standard, and the accuracy rate, false positive and false negative rates, sensitivity, and specificity of PA screening were calculated and evaluated. Quantitative data were tested two-sided by Student's $t$ test. If normal distribution was not met, the Mann-Whitney $U$ test was used. Qualitative data were tested by the nonparametric test. $p<0.05$ was defined as statistically significant difference. The area under receiver operating characteristic curve (AUC) and Youden index were used to assess the diagnostic efficiency. The Youden index was defined as sensitivity plus specificity minus 1 .

\section{Results}

3.1. Baseline Characteristics. Between October 2018 and March 2019, a total of 250 patients visited the hospital due to refractory hypertension. Among them, 5 patients failed to obtain complete ADRR in upright position, and 7 patients left hospital without definite diagnosis. 238 cases met our requirements at the end. Baseline characteristics of the rest 238 patients are shown in Table 1. Age and gender compositions between PA and non-PA groups had no statistical difference. $24.37 \%$ cases were clinically diagnosed as PA. Aldosterone and renin concentrations and ADRR were statistically different between two groups, which meant aldosterone concentration and ADRR in the PA group were higher than those in the non-PA group, and the renin concentration was converse. Among 58 PA patients, 24 cases had aldosteronoma only, 11 cases had idiopathic hyperaldosteronism, 9 cases had adrenal cortical hyperplasia only, 2 cases had aldosteronoma and hyperplasia both, and the rest 12 cases were unidentified.

3.2. Screening Efficiency Evaluation. In the 238 cases, 75 cases with ADRR $>28$ were considered as positive in the screening test, and 50 cases were finally diagnosed as PA in the ADRR positive group, while 163 cases were negative in ADRR screening and 8 cases were finally diagnosed as PA in the group, which meant the false positive and negative rates were $13.89 \%(25 / 180)$ and $13.79 \%(8 / 58)$, respectively. The descriptive representation of 8 false negative cases is shown 
TABLE 1: Baseline characteristics.

\begin{tabular}{|c|c|c|}
\hline Variable & Cohort $(n=238)$ & $p$ value \\
\hline \multicolumn{3}{|l|}{ Sample (cases) } \\
\hline PA & 58 & \\
\hline Non-PA & 180 & \\
\hline Gender (cases) & & 0.556 \\
\hline Male & 127 & \\
\hline $\mathrm{PA}$ & 29 & \\
\hline Non-PA & 98 & \\
\hline Female & 111 & \\
\hline PA & 29 & \\
\hline Non-PA & 82 & \\
\hline Age $\left(\right.$ years) ${ }^{\mathrm{a}}$ & & 0.148 \\
\hline $\mathrm{PA}$ & $48.59 \pm 11.25(28-72)$ & \\
\hline Non-PA & $45.83 \pm 15.96(12-86)$ & \\
\hline Total & $46.50 \pm 14.97(12-86)$ & \\
\hline Aldosterone concentration $(\mathrm{pg} / \mathrm{ml})^{\mathrm{b}}$ & & $<0.001$ \\
\hline $\mathrm{PA}$ & $337.35(61.52-2080.67)$ & \\
\hline Non-PA & $219.02(55.78-990.20)$ & \\
\hline Total & $235.66(55.78-2080.67)$ & \\
\hline Renin concentration $(\mathrm{pg} / \mathrm{ml})^{\mathrm{b}}$ & & $<0.001$ \\
\hline $\mathrm{PA}$ & $5.55(0.10-353.70)$ & \\
\hline Non-PA & $18.30(2.10-146.40)$ & \\
\hline Total & $14.20(0.10-353.70)$ & \\
\hline $\mathrm{ADRR}^{\mathrm{b}}$ & & $<0.001$ \\
\hline PA & $70.12(2.83-615.20)$ & \\
\hline Non-PA & $13.36(0.97-149.72)$ & \\
\hline Total & $16.62(0.97-615.20)$ & \\
\hline Creatinine concentration $(\mu \mathrm{mol} / \mathrm{L})^{\mathrm{b}}$ & & 0.415 \\
\hline PA & $73.5(42-741)$ & \\
\hline Non-PA & $71.0(34-1677)$ & \\
\hline Total & $71.0(34-1677)$ & \\
\hline \multicolumn{3}{|l|}{ PA classification (cases) } \\
\hline Aldosteronoma & 24 & \\
\hline Adrenal cortical hyperplasia & 9 & \\
\hline Idiopathic hyperaldosteronism & 11 & \\
\hline Aldosteronoma and adrenal cortical hyperplasia & 2 & \\
\hline Unidentified & 12 & \\
\hline
\end{tabular}

PA: primary aldosteronism; ALD: aldosterone; ADRR: aldosterone-to-renin ratio; ${ }^{a}$ normally distributed data are presented as mean \pm SD (range); ${ }^{b}$ data without normal distribution are given as median (range).

in Table 2. ADRR correctly screened 155 negative and 50 positive cases in this study with an accuracy rate of $86.13 \%$. Diagnostic sensitivity and specificity were $86.21 \%(50 / 58)$ and $86.11 \%(155 / 180)$. AUC of ADRR screening in the upright position was 0.918 (0.874-0.963, 95\% confidence interval) (Figure 1). We also calculated the diagnostic values of different ADRR decision thresholds for PA screening, which are shown in Table 3.

\section{Discussion}

In our previous retrospective study, we found the most effective cutoff value of CLIA-detected ADRR in the upright position was 28 for PA screening, but we did not know its efficacy in actual work. So, we evaluated its efficacy in this prospective study. The number of patients in case and control groups were estimated from the sensitivity and specificity of ADRR screening test calculated in our previous study. But, we found the number of control group could not be calculated, because the specificity was $100 \%$. So, we took
TABle 2: Descriptive representation of 8 false negative cases.

\begin{tabular}{lccccc}
\hline Sex & Age & Aldosterone & Renin & ADRR & Classification \\
\hline Male & 33 & 197.81 & 12.94 & 15.29 & Aldosteronoma \\
Male & 48 & 192.57 & 13.10 & 14.70 & Hyperplasia \\
Male & 50 & 615.38 & 46.60 & 13.21 & Aldosteronoma \\
Female & 56 & 501.82 & 18.40 & 27.27 & Aldosteronoma \\
Male & 38 & 202.10 & 7.80 & 25.91 & Unidentified \\
Female & 28 & 1116.09 & 61.40 & 18.18 & Unidentified \\
Male & 51 & 254.33 & 11.70 & 21.74 & Hyperplasia \\
Male & 42 & 1000.00 & 353.70 & 2.83 & Aldosteronoma \\
\hline
\end{tabular}

those cases with a final non-PA diagnosis as the control group which were collected at the same time as the case group. Finally, we totally had 238 cases in this study. The ratio of PA patient was $24.37 \%$, which is close to the same ratio, $21.9 \%(p=0.434)$, in our previous study [12] and other studies $[14,16]$. Then, we compared baseline characteristics between these two studies. There was no statistical significance in age and gender compositions, which suggests there 


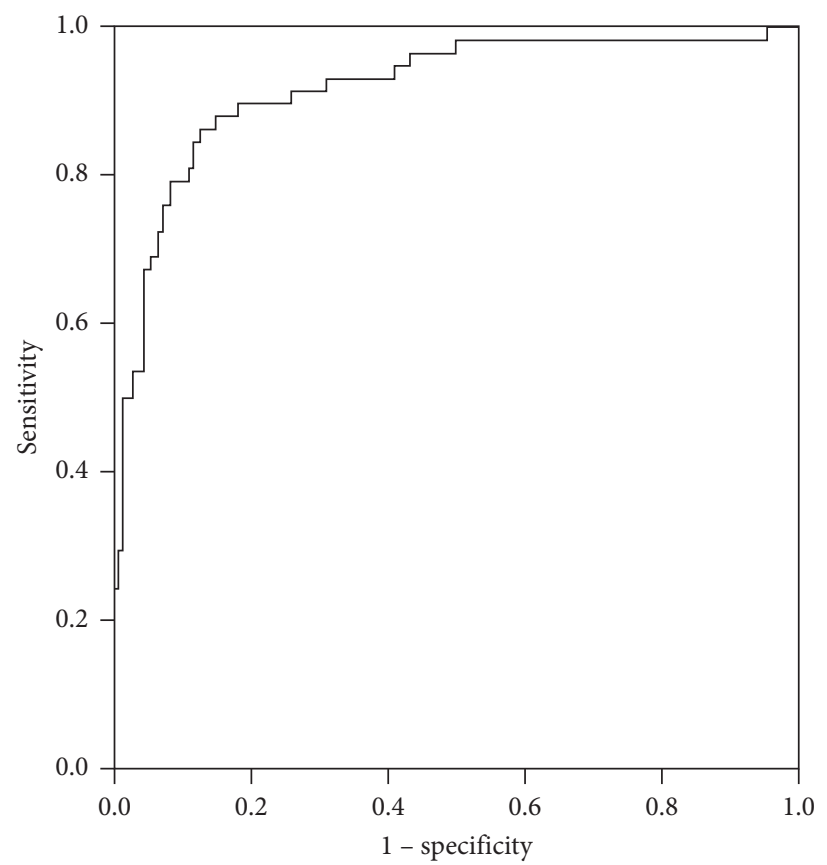

FIGURE 1: Receiver operating characteristic curve of ADRR for PA screening.

TABLE 3: Diagnostic values of different ADRRs for PA screening.

\begin{tabular}{|c|c|c|c|c|c|c|}
\hline ADRR & Accuracy (\%) & False positive rate (\%) & False negative rate (\%) & Sensitivity (\%) & Specificity (\%) & Youden \\
\hline 26.0 & 83.61 & 17.78 & 12.07 & 87.93 & 82.22 & 0.7015 \\
\hline 28.3 & 86.13 & 13.89 & 13.79 & 86.21 & 86.11 & 0.7232 \\
\hline 30.0 & 86.97 & 12.22 & 15.52 & 84.48 & 87.78 & 0.7226 \\
\hline 32.0 & 87.82 & 9.44 & 20.69 & 79.31 & 90.56 & 0.6987 \\
\hline
\end{tabular}

is no significant change in age and gender compositions of refractory hypertension patients to the hospital in recent years $(p=0.065$ and 0.109$)$. But, there was statistical significance in ADRR, aldosterone, and renin concentrations, and the main difference originated from the non-PA group, which meant aldosterone concentration and ADRR were higher in this study, but renin concentration was just the opposite. Raised ADRR in the non-PA group may account for high false positive rate and low specificity, which will be discussed subsequently.

Then, we calculated the accuracy rate. It confirms the high efficiency of ADRR for PA screening in practical work. In this study, we also calculated the sensitivity and specificity rates of ADRR for PA screening. Compared with previous retrospective study, these two rates decreased, especially specificity (100\% vs. $86.11 \%$ ). The lower sensitivity is due to 8 false negative cases. They can be distinguished by high aldosterone concentration (>310 pg/ml in upright position), combined ADRR in the upright and supine positions, images, etc. For example, although it increases 35 false positive cases, high aldosterone concentration recognizes 4 false negative cases in the ADRR negative group. However, the false positive case could be eliminated by many approval tests which are simple, effective. and inexpensive. So, combining ADRR with previous items is a good choice for decreasing the false negative cases. As previously described, high false positive and low specificity partially result from increased ADRR in the non-PA group. However, we do not suggest raising the cutoff value of ADRR for PA screening, because it not only decreases the false positive rate but also obviously increases the false negative rate (Table 3 ). It is not a good choice for PA screening, because PA can be cured. The raised false negative rate which means missing true PA patient will cause serious consequence to PA patient. Besides, the Youden index also reaches maximum when the cutoff value of ADRR is 28 in this study.

However, finding reasons for high false positive rate may help improving the screening efficiency of ADRR. To characterize false positive cases, we compared age and gender distributions between false and true positive cases, but no statistical difference is found ( $p=0.552$ and 0.295 ), which indicates the reason for false positive may be the age and gender of patient. Then, we compared aldosterone and renin concentrations between these two kinds of cases. Although the renin concentration has no difference, the aldosterone concentration is lower in the false positive group, which suggests the false positive rate may be decrease after ADRR screening followed by selecting cases with aldosterone concentration over the reference range. But, unfortunately, it did not work well, because combination not 
only markedly decreased false positive cases (25 vs. 8 ) but also obviously reduced true positive cases (50 vs. 28). Combining ADRR screening with other items may decrease false positive rate, which needs further study.

A special false positive case also gives us some clues. Aldosterone and renin concentrations of this patient in the upright position were detected twice on different days. The first ADRR was over 28, but the second ADRR was less than 28 , actually only 15.3 , without any treatment. The aldosterone concentration was halved at the second test. It suggests that aldosterone may be stimulated or interfered by unidentified reason at the first test. A comparative study may verify our hypothesis. In that study, we compared the aldosterone concentration detected by CLIA and ultraperformance liquid chromatography tandem mass spectrometry. The former is almost all higher than that of the latter, and the correlation coefficient is not very high between them, which suggest CLIA is disturbed by uncertain factors. We need more accurate method to identify the interference and help us modify CLIA detection.

Diabetes is reported to raise the cutoff value of radioimmunoassay detected ARR for PA screening [17]. Then, we assessed the influence of diabetes on screening efficiency in our study. Our results suggest ADRR is influenced by not only diabetes but also impaired glucose tolerance. We found 37 and 13 cases with impaired glucose tolerance in non-PA and PA groups. Although it had no effect on ADRR in PA patients, impaired glucose tolerance elevated ADRR in nonPA patients (median, 17.99 vs. 12.32). If cases with impaired glucose tolerance were excluded, specificity and false positive rate will be improved slightly (Supplementary Table 1). It suggests a special ADRR cutoff value may be needed to screen PA from patient with impaired glucose tolerance. The incidence of impaired glucose tolerance increased in the recent years in China $[18,19]$, which may partially explain the declining efficiency of ADRR for PA screening in this study. Besides, metabolic syndrome is supposed to be related to primary aldosteronism [20]. Effects of other metabolic abnormalities, like hyperuricemia and hyperlipidemia, on ADRR for PA screening may also need assessment.

In this study, we assessed the efficiency of CLIA-detected ADRR for PA screening in newly enrolled patients with refractory hypertension. Overall, it is competent for making accurate primary aldosteronism screening in practical work [21]. But, it still has obvious defects, relatively high false positive and negative rates, which cause a less satisfactory accuracy rate. Further studies are needed to identify reasons and modify the performance of ADRR in PA screening. Finally, our results support the clinical application of CLIAdetected ADRR in PA screening.

\section{Data Availability}

The data used to support the findings of this study are available from the corresponding author upon request.

\section{Conflicts of Interest}

The authors declare that they have no conflicts of interest.

\section{Authors' Contributions}

Wenbin Lin and Yuzhe Li contributed equally to this work.

\section{Acknowledgments}

This work was supported by a horizontal project of the First Affiliated Hospital of Sun Yat-sen University (K0601370).

\section{Supplementary Materials}

Supplementary Table 1: diagnostic values of ARR in different patient sets. (Supplementary Materials)

\section{References}

[1] R. D. Gordon, M. Stowasser, T. J. Tunny, S. A. Klemm, and J. C. Rutherford, "High incidence of primary aldosteronism in 199 patients referred with hypertension," Clinical and Experimental Pharmacology and Physiology, vol. 21, no. 4, pp. 315-318, 1994.

[2] P. Mulatero, M. Stowasser, K.-C. Loh et al., "Increased diagnosis of primary aldosteronism, including surgically correctable forms, in centers from five continents," The Journal of Clinical Endocrinology \& Metabolism, vol. 89, no. 3, pp. 1045-1050, 2004.

[3] G. P. Rossi, G. Bernini, C. Caliumi et al., "A prospective study of the prevalence of primary aldosteronism in 1,125 hypertensive patients," Journal of the American College of Cardiology, vol. 48, no. 11, pp. 2293-2300, 2006.

[4] G. A. Kline, A. P. H. Prebtani, A. A. Leung, and E. L. Schiffrin, "Primary aldosteronism: a common cause of resistant hypertension," Canadian Medical Association Journal, vol. 189, no. 22, pp. E773-E778, 2017.

[5] G. A. Kline, J. L. Pasieka, A. Harvey, B. So, and V. C. Dias, "Medical or surgical therapy for primary aldosteronism: posttreatment follow-up as a surrogate measure of comparative outcomes," Annals of Surgical Oncology, vol. 20, no. 7, pp. 2274-2278, 2013.

[6] J. W. Funder, R. M. Carey, F. Mantero et al., "The management of primary aldosteronism: case detection, diagnosis, and treatment: an endocrine society clinical practice guideline," The Journal of Clinical Endocrinology \& Metabolism, vol. 101, no. 5, pp. 1889-1916, 2016.

[7] J. Manolopoulou, E. Fischer, A. Dietz et al., "Clinical validation for the aldosterone-to-renin ratio and aldosterone suppression testing using simultaneous fully automated chemiluminescence immunoassays," Journal of Hypertension, vol. 33, no. 12, pp. 2500-2511, 2015.

[8] F. H. Perschel, R. Schemer, L. Seiler et al., "Rapid screening test for primary hyperaldosteronism: ratio of plasma aldosterone to renin concentration determined by fully automated chemiluminescence immunoassays," Clinical Chemistry, vol. 50, no. 9, pp. 1650-1655, 2004.

[9] F. Pizzolo, G. Salvagno, B. Caruso et al., "Fully automated chemiluminescence vs RIA aldosterone assay in primary aldosteronism work-up," Journal of Human Hypertension, vol. 31, no. 12, pp. 826-830, 2017.

[10] R. Morimoto, Y. Ono, Y. Tezuka et al., "Rapid screening of primary aldosteronism by a novel chemiluminescent immunoassay," Hypertension, vol. 70, no. 2, pp. 334-341, 2017.

[11] J. Burrello, S. Monticone, F. Buffolo et al., "Diagnostic accuracy of aldosterone and renin measurement by chemiluminescent immunoassay and radioimmunoassay in primary 
aldosteronism," Journal of Hypertension, vol. 34, no. 5, pp. 920-927, 2016.

[12] W. Gan, W. Lin, J. Ouyang et al., "High efficiency of the aldosterone-to-renin ratio in precisely detecting primary aldosteronism," Journal of Human Hypertension, vol. 33, no. 1, pp. 57-61, 2019.

[13] P. Glinicki, W. Jeske, L. Bednarek-Papierska et al., "The ratios of aldosterone/plasma renin activity (ARR) versus aldosterone/direct renin concentration (ADRR)," Journal of the Renin-Angiotensin-Aldosterone System, vol. 16, no. 4, pp. 1298-1305, 2015.

[14] S. C. Käyser, T. Dekkers, H. J. Groenewoud et al., "Study heterogeneity and estimation of prevalence of primary aldosteronism: a systematic review and meta-regression analysis," The Journal of Clinical Endocrinology \& Metabolism, vol. 101, no. 7, pp. 2826-2835, 2016.

[15] M. Ducher, C. Mounier-Véhier, J.-P. Baguet et al., “Aldosterone-to-renin ratio for diagnosing aldosterone-producing adenoma: a multicentre study," Archives of Cardiovascular Diseases, vol. 105, no. 12, pp. 623-630, 2012.

[16] M. Nishizaka, M. Prattubunama, M. Zaman, S. Cofield, and D. Calhoun, "Validity of plasma aldosterone-to-renin activity ratio in African American and white subjects with resistant hypertension," American Journal of Hypertension, vol. 18, no. 6, pp. 805-812, 2005.

[17] C. H. Chang, Y. H. Hu, K. H. Huang et al., "Higher screening aldosterone to renin ratio in primary aldosteronism patients with diabetes mellitus," Endocrine Practice, vol. 7, no. 10, 2018.

[18] J. Shen, A. Goyal, and L. Sperling, "The emerging epidemic of obesity, diabetes, and the metabolic syndrome in China," Endocrine Practice, vol. 2012, p. 178675, 2012.

[19] X. Shen, A. Vaidya, S. Wu, and X. Gao, "The diabetes epidemic in China: an integrated review of national surveys," Endocrine Practice, vol. 22, no. 9, pp. 1119-1129, 2016.

[20] F. Fallo, C. Pilon, and R. Urbanet, "Primary aldosteronism and metabolic syndrome," Hormone and Metabolic Research, vol. 44, no. 03, pp. 208-214, 2012.

[21] P. M. O'Shea, T. P. Griffin, S. Denieffe, and M. C. Fitzgibbon, "The aldosterone to renin ratio in the diagnosis of primary aldosteronism: promises and challenges," International Journal of Clinical Practice, vol. 73, no. 7, 2019. 